\title{
Which is the Future for Organic Monolithic Composite Materials for Analytical Applications?
}

\author{
Ancuta Moga, Héctor Martínez Pérez-Cejuela and Enrique J Carrasco-Correa* \\ Department of Analytical Chemistry, University of Valencia, Spain
}

Submitted: June 04, 2020; Published: July 14, 2020

*Corresponding author: Enrique Javier Carrasco Correa, Department of Analytical Chemistry, University of Valencia, Spain

\begin{abstract}
The quest for methodologies which allows the quantification of low amounts of analytes in complicated matrices, the improvement in efficiency and reduction of costs have been a real challenge in the last years. In this sense, the preparation of novel materials in separation and sample preparation have been widely developed. Particularly, the appearance of porous organic polymers in the latest $80 \mathrm{~s}$ and its more recently combination with other materials from different nature have offer a perfect opportunity to overcome the limitation of conventional silica particles. In this manuscript, a short state-of-the art combined with a viewpoint of the porous organic polymer composites and the possible direction that should be taken in the near future have been considered.
\end{abstract}

Keywords: Future; Organic monolithic composite; Materials; Analytical applications; Chemistry; Polymerization; Novel materials; Crosslinked structure; Monolithic materials

\section{Opinion}

In analytical chemistry, the relentless quest to improve analytical methods in terms of efficiency, speed and cost has provided the scientific world a wide variety of novel materials. Particularly, porous organic polymers (POPs), which were used for the first time in analytical applications by Hjerten et al. [1], are interesting materials that have revolutionized the analytical chemistry field and still have a great capacity to continue providing new ways of being used. POPs are built by radical polymerization using organic building blocks which led to a crosslinked structure united by covalent bonds. A huge number of different POPs have been studied by selecting the adequate precursors (monomers, cross-linkers and initiators) and porogens, although there still infinite combinations to continuous creating novel monolithic materials. The final structure can be tailored using different polymerization initiation modes (chemical, thermal or UV) and mixture compositions. The morphological structure of a typical organic porous bed showing the characteristic microporous and globules is displayed in (Figure 1). Since their first synthesis for analytical applications, this class of materials have been attracted the interest of the researchers due to their fascinating properties such as good permeability and low backpressure [2]. In this sense, their use in chromatographic and sample preparation techniques

overcome the problems that occur with their conventional materials (e.g. silica particles) including irregular packing and poor thermal and chemical stability. However, the major disadvantage of these type of materials is their low surface areas, due to the absence of mesoporous, making difficult the interactions with small molecules [3].

To beat the abovementioned drawback, some strategies have been proposed by the scientific community. Particularly, the most popular is based on the hybridation of materials from different nature resulting in a new one (composite), which combine the best features of both. Among the different strategies to manufacture organic monolithic based composites, the successful approaches introduce nano- or micro-particles taking advantage of the easy tuneable surface on the porous bed and its easy insitu preparation. Regarding the possibilities of combination to construct the hybrid material, the number is enormous since the variety of materials exceed the limits of imagination/science. Some important and recent discoveries have been realized in monolithbased composites when combination with other smart materials as graphene, metal organic frameworks, carbon-nanotubes, metal nanoparticles and macromolecules (for instance: pepsin, trypsin, aptamers and proteins among others) have been performed [4]. However, the real characteristics of these composite materials should be demonstrated by real applications and in this sense, hybrid materials have been extensively used in separation and sample preparation. 


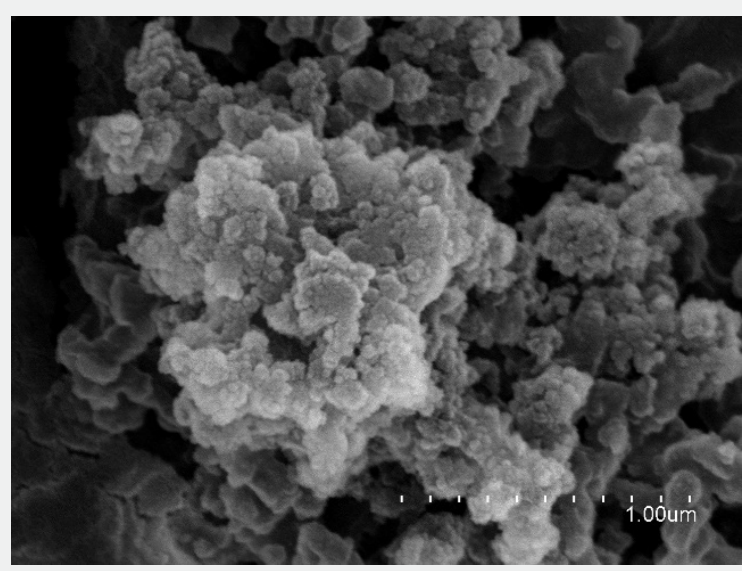

Figure 1: Scanning Electron Microscopy Image of the Characteristic Morphological Structure of a Common POP.

Specifically, the use of POP composites in separation and sample preparation techniques for a wide variety of analytes (antibiotics, pollutants, proteins, hormones among others) have drastically grow up in the last years. Several separation techniques have been selected to study novel monolithic composites as stationary phases. In this sense, conventional high-performance liquid chromatography is the most widely used, although capillary electrochromatography, gas chromatography and capillary and nano liquid chromatography have been also reported. The advantages of monoliths are that can be prepared in situ in the confines of the support and can be covalently attached avoiding the undesirable use of retaining frits, usually used in conventional silica particles stationary phases. In addition, POP composites have been also commonly used for solid-phase extraction (SPE) applications [5]. SPE is usually performed using polypropylene cartridges, but the use of POP composites allows the implementation of new formats like disks, pipette tips and small columns among others. In addition, the use of POP composites allows to perform SPE drawbacks, such as time-consuming, amount of solvent and others. In this sense, POP hybrid materials can be adapted to any other sample preparation procedures as micro-solid phase extraction ( $\mu$-SPE), dispersive solid-phase extraction (d-SPE), magnetic solid-phase extraction (m-SPE), online SPE and will be able to be adapted to any imaginable shape and mode in the future.

In both cases, many composites using metal organic frameworks, nanoparticles and other smart materials have been used for both techniques [6-8]. In general, improvements in the separation or extraction performance is observed due to the increase of mass transfer compared to the pristine material. However, these POP composites still need more improvements to be comparable to the conventional silica particles. Although, some interesting works provided even better results than the conventional counterpart materials. Nevertheless, the lack of drastically increased features in POP composites, usually is due to the incorporation approach (embedding, Figure 2A). Therefore, the increase in other incorporation approaches (copolymerization, Figure 2B and/or surface functionalization, Figure 2C) should be more developed in the next years (Figure 2).

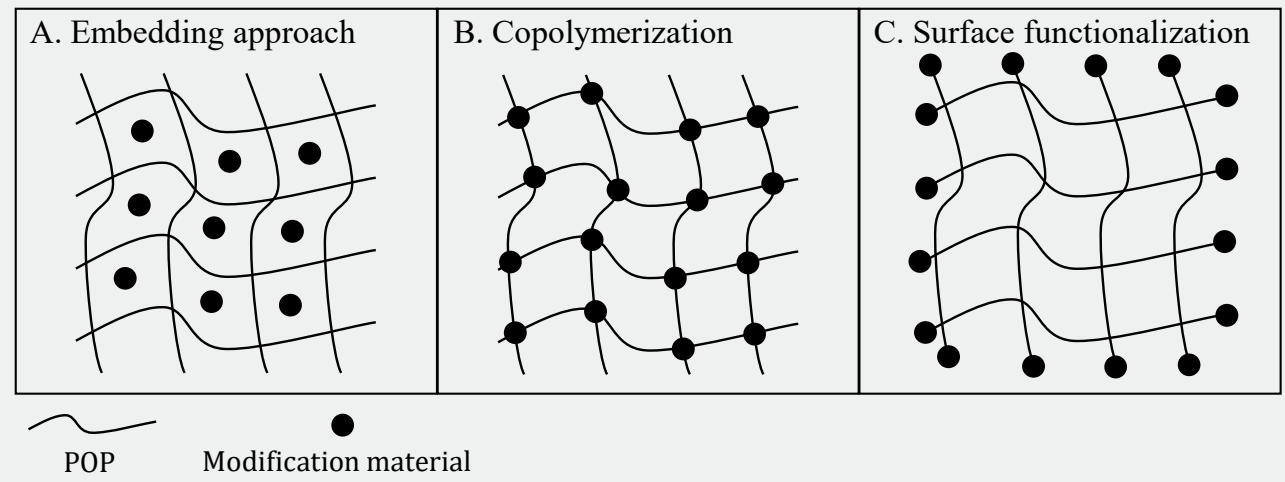

Figure 2: Different Modification Approaches for Preparation of POP Composites. 
Despite the advances performed in the last years in the synthesis on novel hybrid POP materials and the subsequent application in separation and sample preparation techniques, is still a challenge. Within this context, innovative technologies such as the 3D printing can provide a perfect tandem to exploit the advantages of hybrid POPs since these materials can be prepared in any imaginable support structure. However, improvements in the preparation of POP composites on 3D printing and improvement in $3 \mathrm{D}$ technologies should be beat by the scientific community in the next years to provide a new family of 3D printed POP composites that will be in position to fight the conventional silica materials. In addition, another interesting approach is the development of POP composites with (bio) recognition compounds, as aptamers, or molecularly imprinted polymer composites, to obtain methods with high selectivity and specificity that will include the good features of POPs and the smart materials and will offer more costeffective methods compare to the conventional immunoassays, increasing stability and reusability. Nevertheless, the incorporation of aptamers, which are synthetic oligonucleotides, in the surface of POPs or POP composites is still a challenge. Furthermore, the combination of hybrid POPs in methodologies including separation and sample preparation as the use of micro cartridges for SPEonline incorporating separation techniques using POP composites as stationary phases, of its use in microchips and point-of-care devices will be open new avenues in the development of devoted POP composites in the analytical chemistry field.

\section{Acknowledgement}

The authors would like to gratefully acknowledge the financial support from PROMETEO/2016/145 (Consellería de Educación, Investigación, Cultura y Deporte, Generalitat Valenciana, Spain) and RTI2018-095536-B-I00 (MCIU). Héctor Martínez Pérez -Cejuela. Thanks, the support from the Spanish Ministry of Science,
Innovation and Universities for FPU pre-doctoral fellowship. Enrique Javier Carrasco-Correa also thanks the Generalitat Valenciana for a VALi+D postdoctoral research contract (APOSTD/2019/141). The authors extend their appreciation to MCIU for granting the Spanish Network of Excellence in Sample preparation (RED2018-102522-T). This article is based upon work from the Sample Preparation Task Force and Network, supported by the Division of Analytical Chemistry of the European Chemical Society.

\section{References}

1. Hjerten S, Liao JL, Zhang R (1989) High-performance liquid chromatography on continuous polymer beds. J Chromatogr A 473: 273-275.

2. Arrua RD, Causon TJ, Hilder EF (2012) Recent developments and future possibilities for polymer monoliths in separation science. Analyst 137(22): 5179-5189.

3. Urban J (2016) Current trends in the development of porous polymer monoliths for the separation of small molecules. J Sep Sci 39(1): 51-68.

4. Carrasco-Correa EJ, Vergara-Barberán M, Simó-Alfonso EF, HerreroMartínez JM (2019) Smart Materials for Solid-Phase Extraction Applications. Handbook of Smart Materials in Analytical Chemistry, pp. 531-580.

5. Yu M, Wang L, Hu L, Li Y, Luo D, et al. (2019) Recent applications of magnetic composites as extraction adsorbents for determination of environmental pollutants. Trends Anal Chem 119: 115611.

6. Fresco-Cala B, Cardenas S (2018) Potential of nanoparticle-based hybrid monoliths as sorbents in microextraction techniques. Anal Chim Acta 1031: 15-27.

7. Lynch KB, Ren J, Beckner MA, He C, Liu S (2019) Monolith columns for liquid chromatographic separations of intact proteins: A review of recent advances and applications. Anal Chim Acta 1046: 48-68.

8. Connolly D, Currivan S, Paull B (2012) Polymeric monolithic materials modified with nanoparticles for separation and detection of biomolecules: a review. Proteomics 12(19-20): 2904-2917.

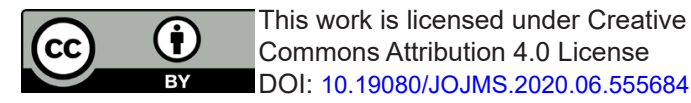

\begin{tabular}{l} 
Your next submission with JuniperPublishers \\
will reach you the below assets \\
- Quality Editorial service \\
- Swift Peer Review \\
- Reprints availability \\
- E-prints Service \\
- Manuscript Podcast for convenient understanding \\
- Global attainment for your research \\
- Manuscript accessibility in different formats \\
( Pdf, E-pub, Full Text, Audio) \\
- Unceasing customer service \\
Track the below URL for one-step submission \\
https://juniperpublishers.com/submit-manuscript.php \\
\hline
\end{tabular}

